\title{
Clinical impact of cardiovascular magnetic resonance with optimized myocardial scar detection in patients with cardiac implantable devices.
}

Anish N Bhuva MBBS ${ }^{\mathrm{a}, \mathrm{b}}$, Peter Kellman $\mathrm{PhD}^{\mathrm{c}}$, Adam Graham MBBS ${ }^{\mathrm{d}}$, Manish Ramlall MBBS $^{\mathrm{a}}$, Redha Boubertakh PhD ${ }^{\mathrm{a}}$, Patricia Feuchter MSc ${ }^{\mathrm{a}}$, Angela Hawkins MSc ${ }^{\mathrm{d}}$, Martin Lowe MD PhD ${ }^{\mathrm{d}}$, Pier D. Lambiase MD PhD ${ }^{\text {b, d }}$, Neha Sekhri MBBS ${ }^{\mathrm{a}}$, Richard J Schilling MD FRCP ${ }^{\mathrm{d}}$, James C Moon MD ${ }^{\mathrm{a}, \mathrm{b}}$, Charlotte H Manisty MBBS PhD ${ }^{\mathrm{a}, \mathrm{b}}$

\section{Affiliations}

a. Department of Cardiovascular Imaging, Barts Heart Centre, St Bartholomew's Hospital, London, UK

b. Institutes for Cardiovascular Science, University College London, London, UK

c. National Institutes of Health, Bethesda, USA

d. Department of Cardiac Electrophysiology, Barts Heart Centre, St Bartholomew's Hospital, London, UK

All authors agree to the following statement:

"This author takes responsibility for all aspects of the reliability and freedom from bias of the data presented and their discussed interpretation"

Abstract word count: 240 words.

Article word count: 3360 words.

Funding: $A B$ is supported by a doctoral research fellowship from the British Heart Foundation (FS/16/46/32187). JCM, PDL and CM are directly and indirectly supported by the University College London Hospitals, National Institute for Health Research Biomedical Research Centre and Biomedical Research Unit at Barts Health NHS Trust.

Competing interests statement: The authors report no relationships that could be construed as a conflict of interest.

\section{Corresponding author:}

Dr Charlotte Manisty,

Department of Cardiac Imaging,

Barts Heart Centre

King George V Building,

West Smithfield,

London EC1A 7BE

United Kingdom

Email: c.manisty@ucl.ac.uk

Telephone: 0044 (0) 2034656115

Fax: 0044 (0)203 4653086

Keywords: pacemaker; defibrillator; cardiovascular magnetic resonance; wideband; late gadolinium enhancement; fibrosis. 


\begin{abstract}
Background: Myocardial scar assessment using late gadolinium enhancement

Cardiovascular Magnetic Resonance (LGE CMR) is commonly indicated for patients with cardiac implantable electronic devices (CIEDs), however metal artifact can degrade images. We evaluated the clinical impact of LGE CMR incorporating a device-dependent metal artifact reduction strategy in patients with CIEDs.
\end{abstract}

Methods: 136 CMR studies were performed in 133 consecutive patients (age $56 \pm 19$ years, 69\% male) with CIEDs (22\% implantable loop recorders [ILRs], 40\% permanent pacemakers [PPMs], 38\% implantable cardioverter defibrillators [ICDs]; 42\% non-MRI conditional) over 2 years, without complication. LGE imaging was tailored to the CIED, using a wideband sequence for left-sided PPMs and ICDs and conventional sequences for ILRs and right-sided PPMs, scoring segmental artifact. Diagnostic utility and impact on clinical management were scored by consensus of experts.

Results: CMR provided unexpected diagnoses in $22(16 \%)$ and changed management in 113 $(83 \%)$ patients. Myocardial scar was present in $92(68 \%)$, with other abnormalities detected in another 13\%. Using conventional LGE, 43 (32\%) studies were non-diagnostic (79\% of defibrillators) compared to $0 \%$ using wideband LGE imaging. Wideband LGE results changed clinical management in an additional 39 (75\%) defibrillator patients and $10(19 \%)$ pacemaker patients when compared to imaging with conventional LGE sequences.

Conclusion: The clinical yield from CMR using optimized LGE sequences in patients with CIEDs is high with no demonstrated clinical risk. A device-dependent LGE imaging strategy using wideband LGE is needed to achieve clinical utility especially in ICD recipients. 


\section{Abbreviations:}

AHA American Heart Association

CRTD cardiac resynchronization therapy defibrillator

CIED cardiac implantable electronic device

CMR cardiovascular magnetic resonance

ICD implantable cardioverter defibrillator

ILR implantable loop recorder

LGE late gadolinium enhancement

MRI magnetic resonance imaging

PPM permanent pacemaker

WB wide bandwidth inversion pulse 


\section{Introduction}

Late gadolinium enhancement (LGE) imaging of myocardial scar using cardiovascular magnetic resonance $(\mathrm{CMR})$ facilitates diagnosis and clinical management across a range of conditions, and provides gold standard non-invasive information regarding scar presence, location, and distribution.[1] This has led to an increase in demand for CMR[2] to determine the aetiology of heart failure,[3] assess viability,[4,5] prognosis,[6,7] and guide both coronary and electrophysiological interventions.[8]

Alongside this rise in CMR, indications for cardiac implantable electronic devices (CIEDs) including permanent pacemakers (PPM) and implantable cardioverter defibrillators (ICD) are expanding, meaning that there are now 2.9 million people in the US alone with CIEDs.[9,10] Many of these patients with electrical abnormalities also have structural cardiac abnormalities, with LGE CMR clinically indicated to accurately assess scar to aid diagnosis and plan electrophysiological procedures.[8] Historically, MRI has been contraindicated in patients with CIEDs due to safety concerns. However, MRI-conditional CIEDs have been developed and are industry standard. Furthermore, it is now known that non-MRI conditional devices can be safely scanned in nearly all circumstances by careful adherence to strict safety protocols.[11,12] These protocols have now been developed with a growing number of centers performing such scanning routinely.

LGE CMR imaging in patients with CIEDs however can be problematic because of metal artifact, also a problem with endovascular aortic repairs (EVAR - present in 50,000 US patients).[13] This limits the clinical yield, with many images rendered non-diagnostic, particularly in patients with defibrillators.[14-17] The effect of larger cardiac resynchronization therapy defibrillators (CRTD) and MRI-conditionality on device artifact have not been studied. 
Recently, a new metal artifact reduction strategy has been developed to reduce artifact on LGE images, using a wideband inversion pre-pulse.[18,19] Although wideband LGE has been shown to be effective at reducing image artifact in defibrillator patients, the clinical benefit of LGE imaging in patients with CIEDs remains unreported - largely due to historical issues related to accessing scans and image artifact. We studied the impact of LGE CMR for diagnosis and clinical management in patients with CIEDs, and measured the incremental value of a device-dependent LGE sequence selection strategy incorporating wideband LGE. 


\section{Methods}

\section{Patient cohort}

Consecutive clinical requests for CMR in patients with CIEDs (implantable loop recorder (ILR), PPM, ICD, CRTD) at a UK tertiary referral center (Barts Heart Centre, London) were recruited. Prior to referral, all patients underwent clinical evaluation, ECG and transthoracic echocardiography and additional investigations where appropriate. Our standard protocol for device scanning involves an initial safety and clinical assessment by a team with both CMR and pacing experience (here led by attending physician CM with dual International Board of Heart Rhythm Examiners and European Association of Cardiovascular Imaging level 3 CMR accreditation). All patients with MRI-conditional CIEDs were included, and patients with non-MRI conditional devices who fulfilled local operating procedure criteria (based on Magnasafe Registry and ESC Guidelines)[11,20] were included after formal informed written consent. Patients with devices implanted prior to 2001 were excluded, as were pacingdependent patients with ICDs where the anti-tachycardia therapies could not be disabled whilst maintaining asynchronous pacing.

All patients underwent device interrogation and re-programming immediately prior to imaging, according to manufacturer and/or ESC guidance (Supplementary Appendix 1).[20] Devices were re-interrogated immediately after the scan and programmed back to the original settings. Adverse clinical outcomes and significant lead parameter changes were noted according to MagnaSafe Registry criteria for significant changes.[11] Patients were subsequently followed up in the device clinic within 3 months if significant changes were noted.

\section{MRI protocol}

CMR was performed at $1.5 \mathrm{~T}$ (Siemens Aera) with a 30 channel phased array receiver coil at Normal Operating Mode (SAR limit $<2 \mathrm{~W} / \mathrm{kg}$ ) with continuous monitoring (ECG and pulse 
oximetry) and verbal contact with patients. The protocol was pre-specified according to the clinical indication and to minimize time spent by the patient within the static/gradient magnetic field. A device-dependent sequence selection strategy was made for cine and perfusion imaging as described elsewhere.[21] T2-oedema (STIR) imaging was performed if myocardial inflammation was suspected. Double inversion recovery T1-weighted fast spin echo (with and without fat suppression) was performed if intra-myocardial fat or pericardial disease were suspected.

LGE images were acquired 5- 20 minutes after administration of $0.1 \mathrm{mmol} / \mathrm{kg}$ of Dotarem (Guerbet S.A., Paris). Sequence selection followed a device- dependent strategy. For all patients with ILRs and right-sided PPMs where artifact was not felt likely to impact on image quality, conventional FLASH (fast low angle shot) LGE imaging was initially acquired. If the device produced artifact (judged by the radiographer), a corresponding wideband LGE sequence was acquired. For all patients with left-sided PPMs and defibrillators, a wideband LGE sequence was acquired in all orientations. This was paired in two long-axis and three short-axis orientations with conventional FLASH (fast low angle shot) LGE, with the same phase encoding direction and field of view and TI adjusted appropriately. A center frequency shift was applied if artifact remained significant. If there were breath-holding difficulties, a paired conventional free-breathing steady-state free precession (MOCO-SSFP) LGE sequence was used at the radiographer's discretion.[22] Typical sequence parameters of the LGE sequences can be found in Supplemental Table 1.

\section{Image analysis}

All LGE images were analyzed for artifact by an observer with greater than four years' experience (AB). Each LGE image orientation was graded for AHA segment artifact and for overall quality on a scale of 0 - 4 , where Grade 0 corresponded to images with no artifact over the myocardium, and Grade 4 corresponded to images where the entire myocardium was obscured by artifact as outlined in Supplemental Figure 1. Scans with a Grade of 3 and 4 
(artifact covering greater than half, or the entire myocardium averaged across all orientations) were classified as 'non-diagnostic'.

\section{Inter-observer reproducibility of artifact grading}

Inter-observer agreement for AHA segment grading was repeated by two senior observers (with greater than 15 and 10 years' CMR experience respectively) for a randomly selected sample of 15 patients with 420 segments ( 2 long axis and 3 short axis orientations per patient), stratified by device type. There was exact agreement in 97 and $95 \%$ of segments respectively.

\section{Assessment of impact on diagnosis and patient management}

Conventional and wideband LGE images were assessed independently by two observers (AB, MR) for significant clinical impact; both for diagnosis and changes to clinical management. A third independent observer $(\mathrm{CM})$ adjudicated any discrepancy between the interpreters. Definitions were pre-defined according to previously published criteria,[23] with data collection from patients' medical records. Final diagnosis was determined by the referring specialist, and we adjudicated a 'new diagnosis' if this final diagnosis was principally determined by the CMR findings, and was not suspected from investigations prior to the CMR. A "change in management" was noted if one of the following criteria were met:

- findings led directly to the guidance of or avoidance of an invasive procedure,

- findings led to the initiation or discontinuation of medication including anticoagulation,

- findings directly resulted in hospital/ clinic admission or discharge,

- findings led to planned genetic testing of the proband or cascade family screening. 


\section{Statistical Analysis}

Data distribution was assessed by histogram and using Shapiro-Wilk test for normality. Continuous variables are summarized as mean \pm standard deviation or median (interquartile range); and categorical variables are summarized as absolute numbers and frequencies. For paired normally distributed lead parameter data, the Student's $t$ test was used. For nonnormally distributed variable analysis, the Mann Whitney test was used. For differences in categorical data, the $C h i$ squared was used. Analysis was performed with Stata statistical software (StataCorp. 2015. Stata Statistical Software: Release 14. College Station, TX: StataCorp LP). All tests were two tailed, and $\mathrm{p}<0.05$ was considered significant. 


\section{Results}

\section{Patient characteristics}

Over two years from December 2015, 136 scans were performed in 133 patients referred consecutively for CMR, age 56 \pm 19 years, $69 \%$ male, $18 \%$ current hospital in-patients, detailed in Table 1. Implanted devices were 40\% PPMs (3 CRT pacemakers), 26\% ICDs, $22 \%$ ILRs, $12 \%$ CRTDs (one also with endovascular aortic repair stent graft). $42 \%$ of pulse generators were non-MRI conditional, and $35(33 \%)$ patients were pacing-dependent of whom seven had defibrillators. 19 (14\%) patients had atrial fibrillation. Before CMR scanning, 29\% had undergone invasive coronary angiography, $14 \%$ cardiac computed tomography coronary angiography (CTCA), 5\% positron emission tomography (PET-CT), $5 \%$ nuclear myocardial perfusion scanning, $2 \%$ exercise stress echocardiography and $12 \%$ of patients had had CMR scans prior to device implantation with the follow-up scans requested to assess for disease progression.

\section{Indication}

Scans were performed most commonly for cardiomyopathy assessment or follow up $(n=47$, $35 \%)$, scar assessment prior to ventricular tachycardia ablation $(n=27,20 \%)$, or assessment of viability $+/$ - ischemia $=15(11 \%)$, Table 1. Scans to investigate an underlying cardiomyopathy were for patients with conduction abnormalities or ventricular arrhythmia with either other abnormal clinical findings or a significant family history.

\section{Impact of CMR on new diagnosis and patient management}

CMR resulted in unexpected new diagnoses in $22(16 \%)$ and changed clinical management in $113(83 \%)$ studies (Table 2).

The most frequent (32\%) new diagnosis was myocardial infarction based on typical scar pattern on LGE images that had not been previously suspected from coronary angiography or 
echocardiography. Left ventricular thrombus was discovered in a further 6 patients not seen on previous echocardiography, all prior to ventricular tachycardia (VT) ablation (22\% of these referrals). CMR led to a new diagnosis of cardiac sarcoidosis in 3 patients (14\%) with no prior diagnosis of extra-cardiac sarcoid, after LGE strongly suggested cardiac involvement, supported by subsequent PET-CT or biopsy. Patients who were diagnosed with dilated cardiomyopathy or myocarditis (14\%) showed mid-myocardial or epicardial LGE patterns in combination with clinical findings. One patient with arrhythmogenic right ventricular cardiomyopathy (ARVC) was diagnosed after RV wall motion abnormalities and dilatation were noted on CMR (not previously seen on echocardiography) in combination with non-imaging Task Force criteria. CMR findings diagnosed hypertrophic cardiomyopathy based on asymmetrical hypertrophy and characteristic scar pattern in a patient with a prior myocardial infarction and presumed ischemic cardiomyopathy. Another patient was diagnosed with an intra-cardiac mass that had not been detected on transthoracic echocardiography.

Impact on clinical management was greatest in the cohort of patients referred for CMR prior to ventricular tachycardia (VT) ablation for scar localization $(n=27,17$ presumed ischemic cardiomyopathy). Ablation strategy was changed in $9(33 \%)$ patients after discovery of left ventricular (LV) thrombus, or selecting an epicardial/ right ventricular approach in nonischemic cardiomyopathy, Figure $1.5(4 \%)$ patients underwent device upgrade (from a pacemaker to a defibrillator) or revision following scar detection as part of risk stratification for sudden cardiac death in patients with non-cardiac sarcoidosis, or a new diagnosis of ARVC. $2(1 \%)$ patients underwent percutaneous coronary intervention or coronary artery bypass grafting after LGE imaging demonstrated myocardial viability. 1 patient with complete heart block underwent right ventricular myocardial biopsy after LGE imaging demonstrated non-ischemic scar suggesting sarcoidosis. Medication management was also influenced, most commonly by commencing antiplatelet therapy $(n=13,10 \%)$ after diagnosis 
of unsuspected myocardial infarction, or by modifying dual antiplatelet therapy with systemic anticoagulation after confirming LV thrombus in patients with known coronary artery disease. In 8 (6\%) patients with suspected or known cardiomyopathies, findings suggested ARVC, hypertrophic cardiomyopathy or Fabry disease, thereby prompting genetic testing and/or family screening.

\section{Late gadolinium enhancement imaging}

All patients underwent late gadolinium enhancement imaging. 3 studies required conventional MOCO-SSFP LGE imaging due to breath-hold difficulty. 9 ILR and 2 rightsided PPM patients had artifact on conventional LGE, and consequently wideband LGE images were acquired following the FLASH LGE sequences. In 10 patients (4 CRTD, 6 ICD patients) with very extensive artifact noted on initial long axis conventional LGE imaging, conventional LGE images were not acquired in all orientations due to futility. Complete myocardial coverage was achieved with wideband LGE sequences as per protocol. Scar was present in $92(68 \%)$ patients.

\section{Artifact distribution, extent and device type with conventional LGE}

Artifact was more common in patients with defibrillators and in the anterior wall, Supplemental Figure 2. With ILR, PPM, ICD and CRTDs, the proportion of completely or almost completely diagnostic scans (Grades $0-1$ ) was $87 \%, 84 \%, 11 \%$, and $13 \%$ respectively. 4 patients with ILRs had artifact covering up to half of the ventricular myocardium, all of whom had older, non-injectable devices. 2 patients with PPMs had non-diagnostic imaging, including one CRT. 4 patients with defibrillators had no artifact, including 3 right-sided generator implants. There was no significant difference between ICD and CRTD-related $\operatorname{artifact}(78 \%$ and $81 \%$ non-diagnostic respectively, $\mathrm{p}=0.83)$.

\section{MRI conditionality and artifact with conventional LGE}


Of 33 studies in patients with left-sided ICDs, 19 (58\%) had non-MRI conditional devices. There was similar artifact with non-MRI conditional compared to conditional ICDs; 75 (59$90) \%$ versus $73(55-79) \%$ segments with artifact, $p=0.4$. The rate of completely nondiagnostic scans was however higher in non-MRI conditional devices (68\% Grade 4, $16 \%$ Grade 3, $16 \%$ Grade 2 in those with non-MRI conditional devices; MRI-conditional ICDs: $14 \%$ Grade 4, 64\% Grade 3, 7\% Grade 2, 14\% Grade 1; $<<0.004)$.

\section{Image artifact using wideband LGE}

Of $43(32 \%)$ studies with non-diagnostic (Grades 3-4) images acquired using conventional LGE, wideband LGE sequences permitted acquisition of diagnostic images (Grades 0-2) in all subjects. Wideband LGE completely or almost completely removed artifact (Grade 0 or 1) in $39(91 \%)$, with residual artifact covering less than half of the ventricular myocardium in the remaining $4(\mathrm{p}<0.001)$, Supplemental Figures 2 and 3.

In the $36(26 \%)$ remaining studies with mild artifact on images acquired using conventional LGE (Grades 1-2), wideband LGE completely removed artifact in $31(86 \%)$ patients, with Grade 1 residual artifact in 5 patients.

\section{Incremental impact on new diagnosis and patient management using wideband LGE}

Using only conventional LGE imaging, new diagnoses were made in $12(9 \%)$ studies, and using wideband LGE new diagnoses were made in an additional 10 (7\%) studies, which were in patients with defibrillators.

Using only conventional LGE imaging, clinical management was changed in 63 (46\%) studies, and using wideband LGE clinical management was changed in an additional 50 (37\%) studies. This incremental benefit was greatest in patients with defibrillators, where the wideband image results directed changes to management in an extra $39(75 \%)$ patients, Figure 2. 


\section{Safety}

There were no clinical complications during or following MRI related to scanning. One selfterminating episode of atrial fibrillation was observed in a patient already under follow up for an atrial arrhythmia. One patient was scanned in a slow stable ventricular tachycardia and two patients were scanned whilst maintaining lidocaine infusion (all pre-ablation for ventricular tachycardia). There was no inappropriate pacing inhibition, unexpected pacing, anti-tachycardia therapy or significant lead parameter changes (See Supplemental Table 2). $45(22 \%)$ individual leads had minor parameter changes, which at follow up of 52 (6-81) days, normalized or were stable within normal limits. 


\section{Discussion}

These data from the largest reported cohort of patients with cardiac implantable electronic devices undergoing CMR highlight the incremental clinical impact of optimized scar assessment. Principally based on LGE findings, CMR identified new diagnoses and/or altered management in five out of every six patients (83\%). Using a device-dependent LGE sequence selection strategy enabled acquisition of diagnostic images in high risk patients within standard clinical workflows, with the greatest incremental clinical impact of a refined wideband sequence in patients with defibrillators.

\section{Clinical utility of myocardial scar imaging using LGE CMR}

Overall, the clinical yield from CMR scans in this cohort was high, with unexpected diagnoses made in $16 \%$ and changes to management in $83 \%$. This is likely to be related to a potentially higher threshold for referral in device patients, and the high prevalence of structural heart disease in this cohort, which may have contributed to the decision for device implantation initially. However, all patients underwent extensive investigations prior to CMR using alternative imaging modalities and/ or invasive investigations. The clinical impact in non-MRI conditional CIEDs, different CIEDs, and in a range of indications has not previously been reported. The clinical utility of LGE CMR is well described, however we show the yield in this cohort of device patients is higher than most previous studies of other patient populations (including heart failure).[23,25-27] Samar et al showed that MRI scans enhance device patient care in $97 \%$ in predominantly non-cardiac indications.[28] Raphael et al showed CMR provided management-changing information in $63 \%$ of patients with MRIconditional pacemakers,[27] the EuroCMR registry showed an impact on patient management in 62\%,[26] and Abbasi et al showed CMR changed management in 52\% of heart failure patients (without CIEDs).[23] 
Probably the greatest clinical impact was related to electrophysiology procedural decisionmaking in patients with VT. Detecting thrombus and localizing ischemic versus nonischemic scar had substantial impact on planned ablation approaches (endocardial vs epicardial and left vs right ventricular), in accordance with previously-published data.[8] In patients with pacemakers, scar assessment guided decisions regarding immunosuppression or device upgrade in sarcoidosis and genetic screening in patients with suspected cardiomyopathy. Across all CIEDs, new diagnoses (principally unexpected myocardial infarction or cardiomyopathies) were made in almost one in five patients.

\section{Incremental clinical value of wideband LGE imaging}

The problem of metal artifact on LGE images in patients with CIEDs is widely appreciated, meaning that CMR scans are often not requested even in patients with MRI-conditional devices when needed. Incorporation of a wider bandwidth radiofrequency inversion pulse has been used by several groups with success to reduce this artifact $[18,19]$ although the incremental clinical utility of this approach over conventional LGE imaging has not been explored. The impact on clinical management was most marked in the defibrillator group where $79 \%$ had non-diagnostic imaging using conventional LGE sequences, but with wideband LGE, diagnostic imaging across the whole group resulted in management changes in $87 \%$. Although previously reported in small numbers,[29] our data suggest that wideband LGE should also be accessible for CMR imaging in patients with PPMs where an additional $19 \%$ of patients had changes to management, when compared to using conventional imaging.

The approach used in this study uses a thin slice 2D wideband (WB) LGE sequence acquired using single shot respiratory motion corrected averaging[22] to improve the signal-to-noise and combined with phase sensitive inversion recovery (PSIR)[30] to optimize myocardial nulling. $23 \%$ of studies were affected by mild or minimal artifact using wideband LGE and all studies were diagnostic. This is similar to recent literature reporting some artifact in $13 \%$ 
and non-diagnostic studies in 3\%.[31] Our approach is robust in the routine clinical environment, enabling scan acquisition even in patients with unstable breathing patterns and susceptibility to arrhythmia. Previous studies[31] reported that $4 \%$ of cases are affected by motion artifact, and hence would benefit from this free-breathing acquisition. Our cohort however was clinically less stable, with $31 \%$ of patients hospitalized or with underlying arrhythmia (including ventricular tachycardia) during scanning, suggesting a greater benefit.

\section{Influences on artifact using LGE imaging}

These data found that there is no appreciable difference in artifact between patients with ICD and CRTDs. Patients with MRI-conditional ICDs had better overall artifact grade, but similar segmental artifact frequency, as compared to those with non-MRI conditional devices. This study was not powered to detect such a difference, and by incorporating a variety of device manufacturers, any effect may have been diluted. Nevertheless, this result may be attributed to the reduced ferromagnetic material content and radiofrequency interference in MRIconditional devices. Despite this improvement, conventional LGE imaging was still nondiagnostic in $78 \%$ of patients with MRI-conditional ICDs.

\section{Safety of CMR in patients with CIEDs}

The combination of increasing evidence supporting LGE CMR for tissue characterisation to aid clinical diagnosis and guide patient management, together with the manufacture of MRIconditional cardiac implantable electronic devices and an expanding body of safety data for imaging non-MRI conditional devices, means that there is a rising demand for myocardial scar imaging using LGE CMR in CIED patients.

This study represents the largest reported cohort of consecutive patients undergoing cardiac MRI with non-MRI conditional cardiac implantable electronic devices, including pacemaker dependent patients with no underlying ventricular rhythm and clinically unwell hospitalized patients with ventricular arrhythmia. Following strict local safety protocols based on ESC and 
HRS recommendations, [20,24] there were no significant clinical complications and no individual lead parameter changes that required intervention, although non-significant, temporary changes were detected.

\section{Service provision}

Despite a growing demand for CMR in CIED patients, predominantly for scar imaging, provision of MRI scanning in this population is currently inadequate.[32] This study highlights the clear clinical utility of CMR in this patient cohort, and should be used to drive service development. The infrastructure required to scan devices is however non-trivial - at our center this involves a radiographer, MRI physicist, cardiac physiologist, and attending cardiologist (for non-MRI conditional devices), with availability of monitoring equipment and an external defibrillator with transcutaneous pacing. This is a similar infrastructure to other previously described safety protocols, $[11,24,25]$ and means that MRI scanning in patients with non-MRI conditional devices is likely to be restricted to specialized centers within healthcare frameworks. A literature is now developing for less intensively monitored scanning protocols[33] in patients with MRI-conditional devices, with recognition by industry of the clinical need for devices designed to require less manual expert input, to improve clinical workflows.[34] 


\section{Study Limitations}

This was a single center study and so reflects the referral patterns and clinical protocols used at our institution. Wideband LGE was not performed in patients in whom conventional LGE provided adequate image quality without artifact and 10 ICD/CRTD patients did not have whole heart conventional LGE due to obvious futility, given the extent of artifact seen on the initial images. The impact of metal artifact on right ventricular scar was not assessed, although RV scar assessment remains problematic even in patients without CIEDs. We did not assess for the incremental benefit of T2 weighted STIR imaging for edema in the study, given that there are currently no optimized sequences available. Scan analysis was not performed blinded because it was evident which sequences were wideband and which were conventional LGE. Image quality analysis and clinical impact was subjective; we used a panel of three observers for clinical impact to minimize this, and applied previously published criteria for determining clinical impact.[23,27] Further studies would help to corroborate our findings. 


\section{Conclusion}

Diagnostic myocardial scar imaging using LGE CMR results in significant changes to clinical management in the majority of CIED patients. A metal artifact reduction strategy using wideband imaging is however often necessary to optimize image quality in both leftsided pacemakers and defibrillator patients. 


\section{References}

[1] Wu E, Judd RM, Vargas JD, Klocke FJ, Bonow RO, Kim RJ. Visualisation of presence, location, and transmural extent of healed Q-wave and non-Q-wave myocardial infarction. Lancet. 2001;357:21-28.

[2] Hendel RC, Patel MR, Kramer CM, Poon M, Hendel R, Carr J et al. Appropriateness Criteria for Cardiac Computed Tomography and Cardiac Magnetic Resonance Imaging. J Am Coll Cardiol. 2006;48(7):1475-1497.

[3] Hundley WG, Bluemke DA, White RD, Woodard PK.

ACCF/ACR/AHA/NASCI/SCMR Expert Consensus Document on Cardiovascular Magnetic Resonance. J Am Coll Cardiol. 2010;55:2614-2662.

[4] Bello D, Shah DJ, Farah GM. Gadolinium Cardiovascular Magnetic Resonance Predicts Reversible Myocardial Dysfunction and Remodeling in Patients With Heart Failure. Circulation. 2003;108:1945-1953.

[5] Kim, R, Wu, E, Rafael, A, Chen, E, Parker, M.A, Simonetti, O, Klocke, et al. The Use of Contrast-Enhanced Magnetic Resonance Imaging to Identify Reversible Myocardial Dysfunction. N. Engl. J. Med. 2000;343:1445-1453.

[6] Gulati A, Jabbour A, Ismail TF, Guha K, Khwaja J, Pennell DJ, Prasad SK. Association of Fibrosis With Mortality and Sudden Cardiac Death in Patients With Nonischemic Dilated Cardiomyopathy. JAMA. 2013;309:896.

[7] Klem I, Weinsaft JW, Bahnson TD, Hegland D, Kim H, Hayes, B. Assessment of Myocardial Scarring Improves Risk Stratification in Patients Evaluated for Cardiac Defibrillator Implantation. J Am Coll Cardiol. 2012;60:408-420. 
[8] Acosta J, Fernández-Armenta J, Penela D. Infarct transmurality as a criterion for firstline endo-epicardial substrate-guided ventricular tachycardia ablation in ischemic cardiomyopathy. Hear Rhythm. 2016;13:85-95.

[9] Nazarian S, Hansford R, Roguin A, Goldsher D, Kamel IR, Calkins H et al. A Prospective Evaluation of a Protocol for Magnetic Resonance Imaging of Patients With Implanted Cardiac Devices. Ann Intern Med. 2011;155:415.

[10] Greenspon AJ, Patel JD, Lau E. Trends in permanent pacemaker implantation from 1993 to 2009. J Am Coll Cardiol. 2012;60:1540-1545.

[11] Russo RJ, Costa HS, Silva PD, Anderson J, Arsha A, Biedermen R et al. Assessing the Risks Associated with MRI in Patients with a Pacemaker or Defibrillator. N Engl J Med. 2017;376:755-764.

[12] Nazarian S, Hansford R., Rahsepar A, Weltin V, McVeigh D, Ipek E et al. Safety of magnetic resonance imaging in patients with implanted cardiovascular devices. $\mathrm{N}$ Engl $\mathbf{J}$ Med. 2017;377:25555-25564.

[13] Dua A, Kuy S, Lee C, Upchurch G, Desai S. Epidemiology of aortic aneurysm repair in the United States from 2000 to 2010. J Vasc Surg. 2014;59:1512-7.

[14] Naehle CP, Kreuz J, Strach K. Safety, feasibility, and diagnostic value of cardiac magnetic resonance imaging in pacemakers and implantable cardioverters/defibrillators at 1.5 T. Am Heart J.2011;161:1096-105.

[15] Sasaki T, Hansford R, Zviman MM. Quantitative assessment of artifacts on cardiac magnetic resonance imaging of patients with pacemakers and implantable cardioverterdefibrillators. Circ Cardiovasc Imaging. 2011;4:662-70. 
[16] Mesubi O, Ahmad G. Impact of ICD artifact burden on late gadolinium enhancement cardiac MR imaging in patients undergoing ventricular tachycardia ablation. Pacing Clin Electrophysiol. 2014;37:1274-83.

[17] Bartels LW, Smits HF, Bakker CJ, Viergever M. MR imaging of vascular stents: effects of susceptibility, flow, and radiofrequency eddy currents. J Vasc Interv Radiol. $2001 ; 12: 365-371$.

[18] Rashid S, Rapacchi S, Vaseghi M, Tung R, Shivkumar K, Finn JP, Hu P. Improved late gadolinium enhancement MR imaging for patients with implanted cardiac devices. Radiology. 2014;270:269-74.

[19] Rashid S, Rapacchi S, Shivkumar K. Modified wideband three-dimensional late gadolinium enhancement MRI for patients with implantable cardiac devices. Magn Reson Med. 2016;75:572-84.

[20] Brignole M, Auricchio A, Baron-Esquivias G, Bordachar P, Boriani G, Breithardt OA et al. 2013 ESC Guidelines on cardiac pacing and cardiac resynchronisation therapy. Eur Heart J. 2013;34:2281-329.

[21] Hilbert S, Jahnke C, Loebe S, Oebel S, Weber A, Spampinato et al. Cardiovascular magnetic resonance imaging in patients with cardiac implantable electronic devices: a devicedependent imaging strategy for improved image quality. Eur Hear J - Cardiovasc Imaging. $2017 ; 3: 1-11$

[22] Kellman P, Larson AC, Hsu L-Y. Motion-corrected free-breathing delayed enhancement imaging of myocardial infarction. Magn Reson Med. 2005;53:194-200.

[23] Abbasi SA, Ertel A. Impact of cardiovascular magnetic resonance on management and clinical decision-making in heart failure patients. J Cardiovasc Magn Reson. 2013;15:89. 
[24] Indik JH, Gimbel JR, Abe H, Teixeira R, Green U, Clarke G et al. 2017 HRS expert consensus statement on magnetic resonance imaging and radiation exposure in patients with cardiovascular implantable electronic devices. Heart Rhythm. 2017;14:e99-e151.

[25] Nazarian S, Roguin A, Zviman MM, Lardo A. Clinical utility and safety of a protocol for noncardiac and cardiac magnetic resonance imaging of patients with permanent pacemakers and implantable-cardioverter defibrillators. Circulation. 2006;114:1277-1284.

[26] Bruder O, Wagner A, Lombardi M, Schwitter J, van Rossum A, Mahrholdt H. The EuroCMR registry. J Cardiovasc Magn Reson. 2013;15:9.

[27] Raphael CE, Vassiliou V, Alpendurada F, Prasad SK, Pennell DJ, Mohiaddin RH. Clinical value of cardiovascular magnetic resonance in patients with MR-conditional pacemakers. Eur Heart J Cardiovasc Imaging. 2015;13:57-57.

[28] Samar H, Yamrozik JA, Williams RB, et al. Diagnostic Value of MRI in PatientsWith Implanted Pacemakers and Implantable Cardioverter-Defibrillators Across a Cross Population. JACC Clin Electrophysiol. 2017;3(9):991-1002.

[29] Hilbert S, Weber A, Nehrke K,Bornert P, Schnackenburg B, Oebel S . Artefact-free late gadolinium enhancement imaging in patients with implanted cardiac devices using a modified broadband sequence : current strategies and results from a real-world patient cohort. 2018;2:1-7.

[30] Kellman P, Arai AE, McVeigh ER, Aletras AH. Phase-sensitive inversion recoveryfor detecting myocardial infarction using gadolinium-delayed hyperenhancement. Magn Reson Med. 2002;47:372-83.

[31] Do DH, Eyvazian V, Bayoneta AJ, Hu P, Finn K, Boyle N. Cardiac magnetic resonance imaging using wideband sequences in patients with nonconditional cardiac 
implanted electronic devices. Hear Rhythm. 2018;15(2):218-225.

[32] Sabzevari K, Oldman J, Herrey AS, Moon JC, Kydd AC, Manisty C. Provision of magnetic resonance imaging for patients with "MR-conditional" cardiac implantable electronic devices: An unmet clinical need. Europace. 2017;19:425-431.

[33] Bertelsen L, Petersen HH, Philbert BT, Svendsen JH, Thomsen C, Vejlstrup N. Safety of magnetic resonance scanning without monitoring of patients with pacemakers. Europace. 2017;5:818-823.

[34] Savouré A, Mechulan A, Burban M, Olivier A, Lazarus A. The Kora Pacemaker is Safe and Effective for Magnetic Resonance Imaging. Clin Med Insights Cardiol. 2015;9:8590. 
Table 1 Baseline Characteristics.

\begin{tabular}{lcl}
\hline Patient Characteristics & \multicolumn{2}{l}{133} \\
\hline \hline$N$ & \multicolumn{2}{c}{$56 \pm 19$} \\
Age/years & 92 & $(69 \%)$ \\
Male & 19 & $(14 \%)$ \\
Atrial Fibrillation & $50 \pm 17$ \\
LV ejection fraction/ \% & 24 & $(18 \%)$ \\
Hospitalized inpatient & \multicolumn{2}{c}{} \\
& \multicolumn{2}{c}{} \\
\hline CMR Indication & 136 \\
\hline \hline$N$ & 47 & $(35 \%)$ \\
Suspected Cardiomyopathy & 11 & $(8 \%)$ \\
Cardiomyopathy follow up & 5 & $(4 \%)$ \\
Isolated complete heart block & 3 & $(2 \%)$ \\
Ejection fraction assessment & 5 & $(4 \%)$ \\
Infiltrative disease & 15 & $(11 \%)$ \\
Ischaemia/Viability & 10 & $(7 \%)$ \\
Myocarditis & 1 & $(1 \%)$ \\
Mass & 5 & $(4 \%)$ \\
Out of hospital VF arrest & 3 & $(2 \%)$ \\
Pericardial disease & 27 & $(20 \%)$ \\
Substrate for arrhythmia & 4 & $(3 \%)$ \\
Thrombus & & \\
& &
\end{tabular}

\begin{tabular}{lrl}
\hline Device Type & & \\
\hline \hline CRT-D * & 16 & $(12 \%)$ \\
EVAR $\dagger$ & 1 & $(1 \%)$ \\
ILR & 30 & $(22 \%)$ \\
ICD $\ddagger$ & 36 & $(27 \%)$ \\
PPM $\S$ & 54 & $(40 \%)$
\end{tabular}

\begin{tabular}{lrl}
\hline Other device details & & \\
\hline \hline Non-MRI conditional & 45 & $(42 \%)$ \\
Pacing-dependent & 35 & $(33 \%)$ \\
Right-sided & 6 & $(6 \%)$ \\
\end{tabular}

\begin{tabular}{lll}
\hline Device Manufacturer & & \\
\hline \hline Boston Scientific & 35 & $(26 \%)$ \\
Biotronik & 17 & $(13 \%)$ \\
Medtronic & 67 & $(49 \%)$ \\
St Jude Medical (Abbott) & 17 & $(13 \%)$
\end{tabular}


Table 1 Baseline Characteristics. Normally distributed variables described as mean and standard deviation. Categorical variables expressed as absolute numbers and percentages.

* 1 patient had an upgrade from ICD to CRT-D between two scans. $\uparrow 1$ patient had an CRT$\mathrm{D}$ and EVAR in situ $\$$ includes one subcutaneous defibrillator $\S$ includes three CRT pacemakers. Three patients were scanned on two occasions.

$P P M=$ permanent pacemaker; $C R T-D=$ Cardiac resynchronization therapy defibrillator; $E V A R=$ Endovascular aortic repair stent graft $; I L R=$ Implantable loop recorder; $I C D=$ Implantable cardioverter defibrillator; PPM= permanent pacemaker 
Table 2 Changes to clinical management by cardiac implantable electronic device.

\begin{tabular}{|c|c|c|c|c|c|c|c|c|c|c|}
\hline \multirow{2}{*}{ Ventricular arrhythmia } & \multicolumn{2}{|c|}{ Total } & \multicolumn{2}{|r|}{ ILR } & \multicolumn{2}{|r|}{ PPM } & \multicolumn{2}{|r|}{ ICD } & \multicolumn{2}{|c|}{ CRT-D } \\
\hline & & & & & & & & & & \\
\hline Guided ablation and substrate mapping & 26 & $(19 \%)$ & & & & & 13 & $(43 \%)$ & 13 & $(36 \%)$ \\
\hline Change in ablation approach & 9 & $(7 \%)$ & & & & & 7 & $(23 \%)$ & 2 & $(6 \%)$ \\
\hline Thrombus detection pre VT ablation & 6 & $(4 \%)$ & & & & & 5 & $(17 \%)$ & 1 & $(3 \%)$ \\
\hline Thrombus exclusion pre VT ablation & 1 & $(1 \%)$ & & & & & 1 & $(3 \%)$ & & \\
\hline Unsuspected infarction in non-ischemic cardiomyopathy & 5 & $(4 \%)$ & & & & & 2 & $(7 \%)$ & 3 & $(8 \%)$ \\
\hline \multicolumn{11}{|l|}{ Device management } \\
\hline Prompted ICD upgrade or device revision & 5 & $(4 \%)$ & & & 5 & $(9 \%)$ & & & & \\
\hline Avoided ICD implantation & 3 & $(2 \%)$ & 2 & $(7 \%)$ & 1 & $(2 \%)$ & & & & \\
\hline \multicolumn{11}{|l|}{ Ischemic heart disease } \\
\hline Investigation of chest pain - prompted angiography/PCI & 2 & $(1 \%)$ & 1 & $(3 \%)$ & 1 & $(2 \%)$ & & & & \\
\hline Investigation of chest pain - avoided angiography/PCI & 14 & $(10 \%)$ & 3 & $(10 \%)$ & 6 & $(11 \%)$ & 5 & $(17 \%)$ & & \\
\hline Led to CABG (+/- aortic valve replacement) & 2 & $(1 \%)$ & 1 & $(3 \%)$ & 1 & $(2 \%)$ & & & & \\
\hline Change in antiplatelet agents & 13 & $(10 \%)$ & 2 & $(7 \%)$ & 3 & $(6 \%)$ & 5 & $(17 \%)$ & 3 & $(8 \%)$ \\
\hline Change in anticoagulation & 6 & $(4 \%)$ & & & & & 6 & $(20 \%)$ & & \\
\hline \multicolumn{11}{|l|}{ Heart failure } \\
\hline Change in heart failure medication & 7 & $(5 \%)$ & 3 & $(10 \%)$ & 2 & $(4 \%)$ & 2 & $(7 \%)$ & & \\
\hline Change in iron chelation therapy & 2 & $(1 \%)$ & & & 2 & $(4 \%)$ & & & & \\
\hline \multicolumn{11}{|l|}{ Cardiomyopathy } \\
\hline Genetic testing/family screening & 8 & $(6 \%)$ & 1 & $(3 \%)$ & 4 & $(7 \%)$ & 2 & $(7 \%)$ & 1 & $(3 \%)$ \\
\hline Immunosuppression for inflammatory cardiomyopathy & 6 & $(4 \%)$ & & & 3 & $(6 \%)$ & 3 & $(10 \%)$ & & \\
\hline Prompted endomyocardial biopsy & 1 & $(1 \%)$ & & & 1 & $(2 \%)$ & & & & \\
\hline Avoided endomyocardial biopsy & 2 & $(1 \%)$ & 1 & $(3 \%)$ & & & 1 & $(3 \%)$ & & \\
\hline Discharge from hospital admission & 7 & $(5 \%)$ & 1 & $(3 \%)$ & 2 & $(4 \%)$ & 3 & $(10 \%)$ & 1 & $(3 \%)$ \\
\hline Discharge from specialist clinic & 23 & $(17 \%)$ & 11 & $(37 \%)$ & 12 & $(22 \%)$ & & & & \\
\hline
\end{tabular}

Table 2 Changes to clinical management by cardiac implantable electronic device. Data expressed as absolute numbers with percentages in parentheses. Percentages add up to greater than $100 \%$ as 19 patients had more than one aspect of management changed. $\mathrm{CABG}=\mathrm{coronary}$ artery bypass graft; CRT-D= Cardiac resynchronization therapy defibrillator; ILR= Implantable loop recorder; ICD= Implantable cardioverter defibrillator; $\mathrm{PCI}=$ percutaneous coronary intervention; $\mathrm{PPM}=$ permanent pacemaker; $\mathrm{VT}=$ ventricular tachycardia. 


\section{Figure Legends}

Figure 1 Case examples of changes to management in patients with cardiac implantable electronic devices using wideband (top row) and conventional (bottom row) late gadolinium enhancement.

Top row: scar imaging using wideband late gadolinium enhancement imaging: (a) Epicardial ablation and anticoagulation after finding an unsuspected large left ventricular thrombus. (b) Secondary prevention medication and targeted endocardial ablation after an occult infarction in a patient with presumed dilated cardiomyopathy. (c) Detailed scar localisation in multiple coronary territories enabling successful ablation. (d) Intervention avoided after establishing a large non viable anterior infarction.

Bottom row: $(\mathrm{e}-\mathrm{h})$ showing paired non-diagnostic conventional FLASH late gadolinium enhancement imaging

$P P M=$ permanent pacemaker $; C D=$ implantable cardioverter defibrillator

Figure 2 Impact of conventional LGE images compared with wideband LGE images on changes to clinical management.

The incremental benefit of wideband LGE was greatest in patients with defibrillators where the sequence improved image quality, leading to changes to clinical management in an additional $75 \%$ of patients. 